\title{
The Recent Highly-Polarized Centimeter Burst in BL Lacertae
}

\author{
H. D. Aller, P. A. Hughes, \& M. F. Aller \\ University of Michigan, Ann Arbor, MI, USA
}

Abstract. Observations with the University of Michigan 26-meter paraboloid at 4.8, 8.0, and $14.5 \mathrm{GHz}$ have been used to test generic shock-based models of the emitting region of BL Lacertae. Large changes in the polarization position angle since 1980 are not consistent with a jet with a simple conical flow.

\section{Introduction}

Figure 1 shows two-week averages of our total flux density and polarization measurements since 1979. A Faraday rotation measure of $-205 \mathrm{rad} \mathrm{m}^{-2}$ (believed to originate in our own galaxy) has been removed from the data. Peaks of 21 outbursts can be identified during the period shown in either the total or polarized flux. At several epochs multiple events are severely blended in our single antenna data, and a number of identified outbursts exhibit complex variations which are probably the result of the close superposition of events. Many of the bursts have been identified with the appearance of new VLBI source components (Denn \& Mutel, these Proceedings, p. 169).

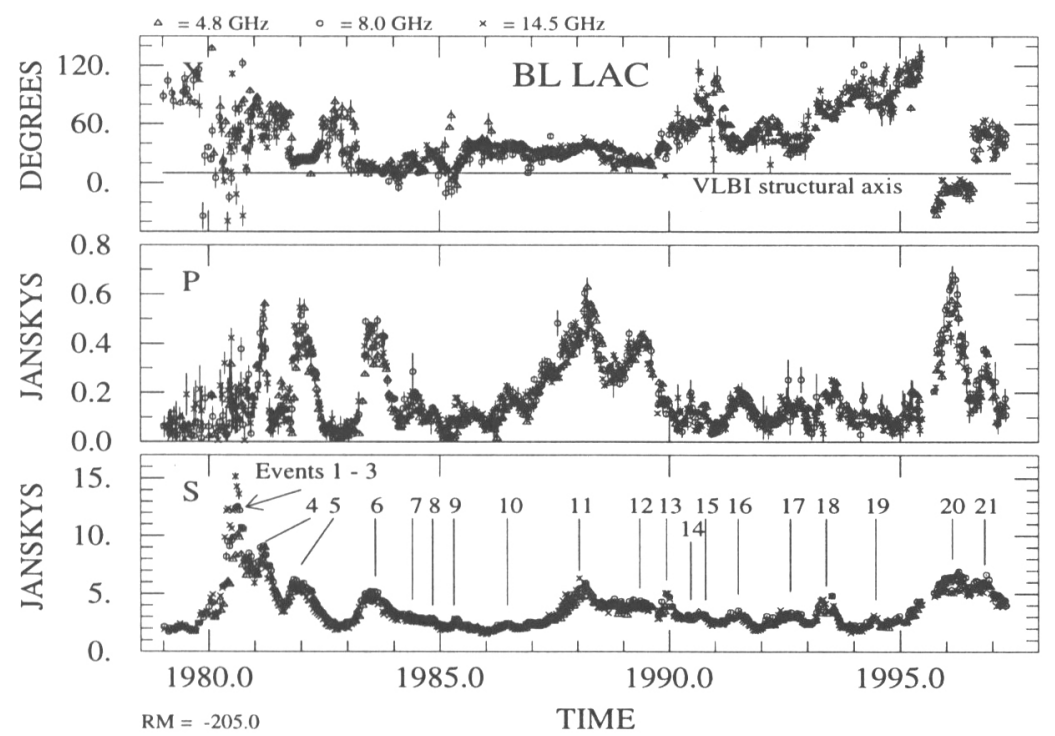

Figure 1. Two-week averages of total flux density, polarized flux and position angle of the electric vector of the polarized emission for BL Lacertae. The horizontal line in the top panel marks the orientation of the VLBI structural axis. Epochs of peaks in identified events are indicated in the bottom panel.

We have now fit five of the bursts by an axial shock model (Hughes, Aller, \& Aller 1989) which uses the compression of a preexisting ambient (primarily random) magnetic field in a relativistic jet flow in order to relate increases in the flux density and the degree of polarization (with the electric vector oriented 
parallel to the axial compression) to the parameters of the shock events. In particular, the time profiles at our three frequencies of the total flux density and polarization can be related to the Lorentz factor of the quiescent flow, the angle of the flow to the line of sight and the strength of the shock.

\section{Outburst Characteristics}

The shock model has been successful in producing a quantitative agreement with some bursts (e.g., 5, 6, 16, \& 20). The inferred Lorentz factors of the quiescent jet flow range from 4.9 to 5.5 and flow angles to the line of sight range from $32^{\circ}$ to $38^{\circ}$. For these bursts we obtained the best fit to the polarization by assuming an axial component of the magnetic field in the quiescent jet flow of $9 \%$ of the random magnetic field, except in burst 21 where an axial field in the quiescent flow of $21 \%$ of the random field was required. Our model does not reproduce the large shift in polarization position angle between 1996 and 1997. The modelfits to these bursts spanning a 15-year period appear surprisingly homogeneous, suggesting little monotonic change in the parameters of the jet flow over the period. However, the model appears to be in qualitative disagreement with other events during the same period (e.g.bursts $13 \& 19$ ). There appear to be two types of bursts: highly polarized events $(P>10 \%)$ with flat spectra, indicative of low internal absorption (e.g.bursts $5,6,16, \& 20$ ) and low polarization events with steep self-absorbed flux spectra (e.g.bursts $1,2, \& 13$ ). We suggest that these self-absorbed events may originate deeper in the core.

Although many of the bursts exhibit polarization position angles near to the average VLBI orientation of $10^{\circ}$, a wide range of polarization orientations is found in other events, suggesting that either there is a range in flow directions (together with significant relativistic aberration effects) or that oblique shocks (not included in our model) often play an important role in the observed emissions. The polarization position angles are distributed very asymmetrically about the structural orientation observed by VLBI. This asymmetry may result from a true curvature in the jet flow structure (such as a helix) as has been suggested in recent VLBI maps (Denn \& Mutel, these Proceedings, p. 169).

In several of the bursts, rapid events, sometimes lasting a month or less, are seen superimposed on what are perceived to be much longer events: examples are bursts 18 and 20 . Interesting question are whether morphological structures can be identified in VLBI maps with these short-term variations and if the structure in the burst profiles are actually the result of multiple short-term events or if these fluctuations result from some type of modulation of the jet flow.

Acknowledgments. We gratefully acknowledge support from NSF grant AST-9421979.

\section{References}

Hughes, P. A., Aller, H. D., \& Aller, M. F. 1989. ApJ, 341, 68-79. 